\title{
THE 3-D DISTRIBUTION OF ABELL CLUSTERS
}

\author{
Will Sutherland \\ Institute of Astronomy, Cambridge CB3 0HA, England.
}

The Struble \& Rood catalogue ( $A p . J . S u p p, 63,543)$ of all measured Abell cluster redshifts is analysed, with corrections for the selection biases. This contains 533 redshifts with $|b| \geq 30^{\circ}, z \leq 0.3$ compared with 104 in the sample of Bahcall \& Soneira $(A p$. J. 270, 20). Although the catalogue contains biases in angular position (redshifts are preferentially measured in apparent "supercluster" regions) the information on redshift clustering is effectively unbiased since one cannot tell a priori whether pairs of clusters close on the sky are really associated in redshift. Thus the distribution of redshift differences for pairs of given angle \& distance classes, $f\left(\Delta z \mid \theta, D_{1}, D_{2}\right)$, is a fair sample of the true distribution. Then by normalising to the "correct" angular correlation function, we obtain the joint distribution $f(\Delta z, \theta)$ and hence $\xi(r)$. In practice, in the estimation of $\xi$ we assign each pair a weight $a$ where

$$
a=\frac{1+w_{A}\left(\theta ; D_{1}, D_{2}\right)}{1+w_{S R}\left(\theta ; D_{1}, D_{2}\right)}
$$

where $w_{A}$ is the correlation or cross correlation for the appropriate subset of the whole Abell catalogue. This gives $\xi(r) \approx\left(r / 20 h^{-1} M p c\right)^{-1.8}$, just slightly smaller than the result of Bahcall \& Soneira, but here $\xi(r) \approx 0$ for $r \geq 50 h^{-1} M p c$.

However, calculating $\xi$ as a function of projected separation $r_{p}$ and redshift separation $r_{z}$, a strong positive tail is found for $r_{p} \leq 20 h^{-1} M p c$, extending to $r_{z} \sim 200 h^{-1} M p c$. This effect is not due to the selection biases as it is present in the nearly complete $D \leq 4, R \geq 0$ subsample. It has been previously noted by Ciardullo, Ford \& Harms $(A p . \bar{J}$. 293, 69). Bahcall, Soneira \& Burgett (Ap. $J ., 311,15)$ claim that this elongation is bounded and infer that it is caused by peculiar velocities $\sim 2000 \mathrm{~km} / \mathrm{s}$. However, although the number of excess pairs does fall at large $r_{z}$, the correlation function (which is excess count divided by random) stays clearly positive, indicating the presence of line-of-sight selection effects in the Abell catalogue.

These projection effects can be compensated for using a method similar to that used by Kruszewski ( $A p$. J., in press) to analyse quasar clustering; after the pair counts are binned in $r_{z}$ and $r_{p}$, columns of constant $r_{p}$ are normalised so that $\overline{\xi\left(r_{z}, r_{p}\right)}=0$ for large $r_{z}$.

This method of analysis gives $\xi(r) \approx\left(r / 12 h^{-1} M p c\right)^{-1.8}$ indicating that the standard $\Omega_{0}=1$ cold dark matter model should not be excluded. 